Itinéraires Itinéraires

Littérature, textes, cultures

2015-3 | 2016

Ethos numériques

\title{
Faire écouter la littérature avec les yeux
}

Variations de l'ethos de l'écrivain dans un environnement numérique : les sites d'Éric Chevillard, de Chloé Delaume et de Régine Detambel

Listening to Literature with Your Eyes. Variations of Writers' Ethos in a Digital Environment: Éric Chevillard, Chloé Delaume and Régine Detambel's Websites

\section{Sylvie Ducas}

\section{OpenEdition}

\section{Journals}

Édition électronique

URL : http://journals.openedition.org/itineraires/3031

DOI : 10.4000/itineraires.3031

ISSN : 2427-920X

Éditeur

Pléiade

Référence électronique

Sylvie Ducas, «Faire écouter la littérature avec les yeux », Itinéraires [En ligne], 2015-3 | 2016, mis en ligne le 01 juillet 2016, consulté le 02 mai 2019. URL : http://journals.openedition.org/itineraires/3031 ; DOI : 10.4000/itineraires.3031

Ce document a été généré automatiquement le 2 mai 2019.

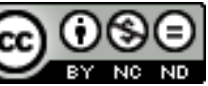

Itinéraires est mis à disposition selon les termes de la licence Creative Commons Attribution - Pas d'Utilisation Commerciale - Pas de Modification 4.0 International. 


\section{Faire écouter la littérature avec les yeux}

Variations de l'ethos de l'écrivain dans un environnement numérique : les sites d'Éric Chevillard, de Chloé Delaume et de Régine Detambel

Listening to Literature with Your Eyes. Variations of Writers' Ethos in a Digital

Environment: Éric Chevillard, Chloé Delaume and Régine Detambel's Websites

\section{Sylvie Ducas}

« Escuchar a los muertos con los ojos ${ }^{1}$. »

Quevedo (1580-1645)

Le vers du poète Quevedo cité en exergue de cette étude évoque les absents qui peuplent le grand cénotaphe de la littérature et que ressuscite l'acte de lire. Rien d'étonnant s'il a déjà servi de fil d'or au propos de l'historien du livre, Roger Chartier, dans une réflexion dont nous ne retiendrons ici que ce qu'elle nous dit des «mutations du présent » et "défis de la textualité numérique " (Chartier 2008). Si l'historien s'interroge sur les bouleversements des "supports de l'écriture, la technique de sa reproduction et de sa dissémination, et [d]es façons de lire " imputables à la révolution numérique, s'il reconnaît que cette dernière « oblige à une radicale révision des gestes et des notions que nous associons à l'écrit» (Ibid.: 20), s'il pointe enfin une rupture décisive dans la conception de la propriété littéraire «dans un monde où les textes sont mobiles, malléables, ouverts », et où chacun peut apporter son opinion et poursuivre la phrase, il ne pose pas la question de la place et des stratégies d'image de l'écrivain dans cet espace numérique dans lequel l'« ordre des discours qui fut toujours un ordre des livres» (Ibid.: 22) est de plus en plus remis en cause, et qui fait violence à l'association traditionnelle entre une autorité auctoriale et la publication qui la couche sur le papier pour qu'elle soit lue.

2 Face à cette violence faite à ses textes, appelés à être donnés à lire et à voir dans des formes et via des réseaux numériques qui n'ont plus grand-chose en commun avec ceux d'autrefois, comment l'écrivain contemporain renégocie-t-il son image et son discours? Comment s'y donne-t-il à voir et à entendre et comment fait-il « écouter avec les yeux » 
ses écrits, et plus largement son idée de la littérature, écrits et conception littéraire jamais loin, on le verra, de l'image auctoriale qu'il se construit en ligne?

On sait que l'ethos participe à la construction de l'identité, qu'elle est cette «stratégie de parole d'un locuteur qui oriente le discours de façon à se façonner à travers lui une certaine identité » (Maingueneau 2002). Dans le cas de l'écrivain, les supports médiatiques et numériques entrent en jeu comme vecteurs de cette construction qui va permettre « de créer un rapport nouveau à soi et à l'autre " (Amossy 2006: 96). Avec l'ethos, ce sont les postures auctoriales que ces réseaux numériques accueillent et/ou engendrent qu'on entend également interroger, en se demandant si le dispositif technologique influence cette image de soi en écrivain, et si oui, comment. On se rappelle que dans la notion de "posture » d'auteur telle que Jérôme Meizoz (2009) l'emploie, deux dimensions sont inséparables: une dimension non discursive renvoyant à l'ensemble des conduites non verbales de présentation de soi, et une dimension discursive qu'il appelle l'ethos discursif. Autrement dit, étudier une posture d'auteur dans le réseau littéraire numérique suppose alors de s'intéresser d'une part à l'aspect comportemental et non verbal de la posture, à savoir la présentation de soi - l'attitude, l'apparence, la conduite au sens weberiensensible dans les photos, visuels et vidéos éventuels mis en ligne, dans les contextes où il incarne sa "fonction-auteur", selon les variations des statuts auctoriaux inscrites dans l'histoire littéraire et «le répertoire historique d'ethos [sic] incorporés, affichés, renversés ou singés » (Meizoz 2007 : 23) ; et d'autre part, prêter attention à la dimension discursive de la posture, l'ethos discursif tel notamment qu'il a déjà pu être étudié, en confrontant les discours sur l'auteur à ceux tenus par l'auteur lui-même.

Peu de travaux ont jusqu'alors été consacrés à la position de l'écrivain face au nouveau contexte technologique du Web 2.0. Avant l'enquête de Cécile Méadel et Nathalie Sonnac (2012a, 2012b) et celle de Valérie Beaudouin (2012), le colloque tenu en 2010 à l'université de Paris Ouest, L'auteur en réseau, les réseaux de l'auteur' ${ }^{2}$,'était interrogé sur la définition de l'auteur à l'ère du numérique et sur la façon dont il pouvait y asseoir une autorité symbolique, selon quelles stratégies et au cœur de quels réseaux (de professionnels, de lecteurs, de pairs, réseaux sociaux). Les contributions avaient permis de pointer quelques grandes tendances : l'usage biface du numérique, comme une vitrine publicitaire ou bien comme un laboratoire d'écriture et de création; la résistance des pratiques traditionnelles de publication associées à l'imprimé; le recyclage de techniques déjà éprouvées en matière de promotion littéraire ; la pérennité de l'imaginaire, des formes et de la culture de l'imprimé. Mais cinq ans plus tard, quelle place l'écrivain occupe-t-il dans cette nébuleuse de discours du littéraire?

5 Les trois écrivains de la présente étude ont délibérément été choisis pour leur engagement non militant ni pionnier sur la toile, de façon à ce que la posture - notamment le portrait de l'écrivain en ingénieur ou en mécano - ne soit pas outrée ni d'emblée dictée par ce parti pris d'un outil numérique trivialisé à trop vouloir le banaliser et montrer qu'on est techniquement compétent. Pas de découvreurs du numérique à la façon d'un François Bon (Deseilligny 2015) ou de Marc Villemain (Chapelain et Fort 2015), donc, mais néanmoins une performeuse (Chloé Delaume) habituée à créer à partir de supports multiples, dont les supports numériques (Ducas 2010, Guillet 2010) et deux écrivains "ordinaires" en ce qu'ils sont nés de la publication imprimée, y restent attachés (Éric Chevillard et Régine Detambel)³. Les trois écrivains retenus n'explorent pas dans leur œuvre les potentialités d'une littérature numérique, dématérialisée, conçue comme exploration expérimentale de l'hypertexte et de l'architexte. En tout cas, ils ne 
s'exhibent pas en experts aguerris du Web; ils sont avant tout des écrivain(e)s ouverts aux possibilités d'outils modernes de communication et d'écriture, et quel que soit leur degré d'implication dans la gestion numérique de leur image publique, ils continuent d'écrire et de publier des livres. Tous les trois ne font pas pour autant partie de ces " écrivains imperturbables" (Bon 2011: 219) indifférents à Internet et que méprisent ceux qui s'en disent les précurseurs: ils ont un site dédié, un d'entre eux un blog, ils communiquent tous plus ou moins via les réseaux sociaux, mais aucun d'entre eux n'est un auteur de best-seller ni ne jouit d'une grande notoriété, de sorte que l'on pourra poser la question de leur ethos numérique sans l'écran d'une peoplisation à la Lévy ou d'une surmédiatisation à la Houellebecq risquant de fausser les perspectives et de gauchir les conclusions.

Dans ces pages, nous défendrons la thèse selon laquelle ces ethe auctoriaux sont affectés par les métamorphoses du geste d'écrire et de se rendre visible qu'induisent les dispositifs numériques, métamorphoses dans lesquelles se reconfigure la définition de la littérature et de l'écrivain.

\section{Ethos public ou ethos publicitaire?}

7 La visite d'un site d'écrivain n'est plus «la visite au grand écrivain " (Nora 1986) d'autrefois. Si elle en garde la dimension topographique et peut-être, pour les plus fans des internautes, quelques vestiges de voyeurisme ou de fétichisme à l'égard d'une image fantasmée du grand écrivain qu'on voudrait à l'écritoire ou dans son cabinet de travail, elle n'est pas cette « visitation » religieuse de l'idole ou du démiurge, pas plus que l'image auctoriale que l'internaute y découvre ne tient de l'apparition sacrale d'un écrivain réduit à la métonymie d'une voix et d'un regard. L'évolution des techniques du son et de l'image et des technologies numériques a transformé le rituel de la visite, mais aussi l'image auctoriale visitée. Si Olivier Nora rappelle qu'avec l'entretien radiophonique et télévisé «le public ne visite plus l'auteur, c'est l'auteur qui fait désormais intrusion dans l'espace familier du public » (Ibid. : 581), on peut dire que la visite d'un site d'écrivain, elle, est une consommation d'image auctoriale qui n'implique pas d'avoir lu une seule ligne de l'auteur, un geste d'information qui tient du «braconnage culturel» (Certeau 1980) plus ou moins hasardeux, dont la motivation va de la simple curiosité au culte médiatique (Le Guern 2002), geste que la technologie numérique autorise à être multiple, cumulatif, itératif, ou bien rester à l'état de trace d'un passage éphémère sur la Toile. La technologie numérique, elle, quand elle prend la forme d'un site d'écrivain ${ }^{4}$, est tributaire du marché de l'offre éditoriale, ce marché risqué (puisqu'il ne répond pas à une demande) auquel l'écrivain participe, et de la péremption des supports qui invite le site à une perpétuelle transformation, réactualisation, non sans incidence sur l'image auctoriale qu'il construit et véhicule. De ce point de vue, Chloé Delaume en est à la troisième refonte de son site entre 2010 et $2015^{5}$ et y gagne de plus en plus une image d'artiste performeuse; le site récent d'Éric Chevillard étoffe ce qui n'était au départ qu'un blog ouvert en septembre 2007, nommé L'Autofictif, dans lequel il écrivait quotidiennement trois petits billets ou fragments de deux à huit lignes, à la façon d'un cabinet d'écriture virtuel; s'y dessine désormais un portrait d'auteur " Azerty $^{6}$ » tout entier dévoué à l'écriture, que symbolise la photographie de machine à écrire sur la page d'accueil et le dessin du crabe sur la page tapuscrite renvoyant à son livre La Nébuleuse du crabe (1993) qui met précisément en scène un personnage nommé Crab, double de papier de l'écrivain. 
8 Mais «l'homme et l'œuvre» qui s'y construisent, entre la vitrine et le monument, n'excluent pas les logiques marchandes qui travaillent tout site. S'il n'est pas qu'un simple catalogue en ligne faisant état de l'avancement d'une production littéraire ni un site d'éditeur mettant en avant une ligne éditoriale et tenant d'un branding fondé sur quelques écrivains-phares pour mieux vendre à Babel, il est malgré tout un dispositif d'autopromotion par lequel l'écrivain cherche à se rendre visible au cœur de la pléthore et de l'hyperchoix et prend la pose, « acteur central de la vie médiatique de son livre et de sa visibilité ultérieure » (Méadel et Sonnac 2012a: 156). En témoignent les portails des trois sites étudiés, affichant chacun à sa manière un écrivain en ordre de parade : une signature en police manuscrite pour Éric Chevillard dans sa posture de lettré, avec un exergue ironique - « Mes livres étaient introuvables. J'ai dû me résoudre à les écrire »prônant clairement la suprématie du geste auctorial de l'écriture sur les supports qui les médiatisent pour cet auteur qui vend peu ; notice bio-bibliographique accompagnée d'une photographie de l'auteure en performeuse lisant son texte sur une scène pour une Chloé Delaume théâtrale et incarnée; dualité des rubriques "écrire/animer" pour Régine Detambel et nom de l'auteure séparé de sa photographie par un bandeau « conférencesateliers-bibliothérapie", comme sur une carte de visite qui la place avant tout en professionnelle, animatrice impliquée dans la vie bibliothécaire qui la fait vivre.

De même, l'incontournable rubrique de la bibliographie semble-t-elle ne plus se contenter de son statut de "liste d'œuvres", au sens où l'œuvre serait l'accomplissement autotélique et téléologique du projet de l'écrivain, liste de références associée à une tout aussi incontournable présentation biographique dont l'ambition est de viser à «aller droit à l'auteur sous le masque du livre », comme disait Sainte-Beuve, et de construire par là même du monument auctorial dont les livres sont le frontispice obligé. Des marqueurs issus du marché éditorial contaminent désormais cet espace de la liste : de façon triviale, ISBN et EAN, ces outils qui aident à simplifier la gestion informatique pour tous les intervenants de la chaîne du livre (imprimeur, éditeur, libraire, bibliothécaire, etc.) figurent dans les mentions légales de chaque ouvrage sur le site d'Éric Chevillard, un feuilleteur numérique, comme chez certains éditeurs ou libraires rompus à l'ebook, permettant d'en lire des extraits, comme si le numérique peinait à sortir des marqueurs du livre qu'il imite pour parler d'un auteur. Toutefois, l'ethos de l'écrivain consacré cherche à se nicher dans ce dispositif commercial où la recommandation n'est plus injonctive et qualitative comme dans la prescription traditionnelle, mais suggestive: il s'affiche, par exemple, dans le déroulé des actualités, par des photographies de l'auteur en couverture d'ouvrages critiques sur son œuvre ou lors de la remise de prix littéraires, autrement dit dans des espaces légitimants.

Chez Régine Detambel, les nouveautés annoncées en facing sur la page d'accueil exhibent, par un clic sur la couverture, le prix des ouvrages, donnent accès par un autre à des documents promotionnels ( $\mathrm{du}$ type quatrième de couverture ou argumentaire commercial, dont Genette (2002) a montré qu'ils constituent des lieux hautement stratégiques en termes de captation marchande et d'impulsion d'achat); une revue de presse exhaustive complète l'accès à des extraits, avec résumé du livre, avis de l'éditeur, dans une présentation qui semble hésiter entre Babelio et Amazon. Mais c'est dans l'avis de l'auteure et sa lecture sonore via des podcasts audio que l'écrivaine tente de développer une posture pleinement littéraire capable de contrebalancer celle de « camelot » qu'on est parfois tenté de lui prêter; et c'est par sa voix qu'elle fait écouter l'écriture dans sa parole, quand des entretiens retranscrits font entendre - même s'il faut 
cette fois lire, à d'autres endroits du site - l'écrivaine explicitant sa démarche d'écriture. De même, dans la liste bibliographique proprement dite, sous le chapeau "Écrire ", concurrent de celui intitulé "Animer », selon une bipolarité qui n'est pas sans rappeler celle des Mots de Sartre, l'écrivaine fait entendre une voix de connivence dans certaines entrées - «À lisotter ", «Billet du lever », «Le grand élucidaire », comme pour mieux colorer de littéraire un espace que l'on ne veut pas que marchand.

11 Seule Chloé Delaume, dans une posture artiste plus radicale qui lui ressemble, issue des collectifs d'artistes indépendants et anticonformistes qu'elle fréquente et qu'elle présente sur son site, semble tout ignorer de ce dispositif commercial et renvoie simplement à des extraits du texte en chantier ou achevé, ou relègue pour mieux les évacuer (comme Éric Chevillard) vers le site de l'éditeur toutes les questions triviales de la marchandise livre et de son achat.

Dans cette nouvelle tribune que représente le site, afficher une image publique de soi revient donc pour l'écrivain(e) à négocier cette image en endossant tant bien que mal un ethos publicitaire lié aux logiques marchandes qui régissent la consommation littéraire aujourd'hui, particulièrement dans l'environnement numérique. Au point que la question que l'on se pose est celle de savoir si l'image de soi est vraiment contrôlée par l'auteur et de son initiative, ou bien si elle est déléguée à un webmaster. S'il semble que Chloé Delaume et Régine Detambel soient les artisans de leur propre mise en scène, la chose est moins certaine pour Éric Chevillard. Toutes deux sont présentes sur Facebook, qu'elles utilisent l'une et l'autre comme un espace de publicité, de médiatisation et d'agenda, elles y emploient le «je » pour décrire, comme dans leur site, leur actualité (publications, conférences, lectures, etc.), sont expertes, pour l'une, des scénographies autofictionnelles et de l'exploitation des supports audiovisuels et numériques (Chloé Delaume) et pour l'autre, de la communication et de l'animation bibliothécaires, y compris dans ses formes numériques (Régine Detambel). Chloé Delaume est la seule à tweeter et à relayer ses tweets sur son site par un ironique « \#cuicuitons ", participant au "gazouillis de Twitter " (Peras 2012). Éric Chevillard, lui, ignore tout des réseaux sociaux, du moins ne les pratique pas, même si son blog, articulé à son site, incite à communiquer sur tous les réseaux et à laisser des commentaires, comme le font tous les éditeurs, libraires et bibliothécaires aujourd'hui. Son ethos distancié, entre posture et dérobade, tient de celui de l'écrivain à l'œuvre et du paradigme de la solitude qui, depuis le romantisme, lui est associé : peu présent en image, absent par la voix, si ce n'est celle, narrative, de son locuteur, burlesque et décalé, que contredisent le sérieux et le classicisme du site, tendant au portail d'information et de documentation. Tout le contraire d'une Chloé Delaume soucieuse d'inviter le visiteur à la fiction collective et collaborative selon des formes discursives homogènes, la voix et le timbre qui se font entendre - «la petite musique », disait Céline - étant les mêmes sur le site ${ }^{7}$ et dans les livres. Dans son cas, c'est plutôt du côté des réseaux sociaux, de la démultiplication des représentations photographiques de soi (abondantes mais jamais les mêmes d'un endroit à l'autre) et de l'hétérogénéité des appellations la désignant que le numérique risquerait d'influer sur l'identité auctoriale et de construire une image dépareillée; si c'est l'unité d'une écrivaine polyvalente et "touche-à-tout", mais d'une écrivaine avant tout dont il s'agit ${ }^{8}$, sur son site elle se disperse également comme éditrice, performeuse, musicienne, chanteuse, réalisatrice, et ce sont les champs de la performance, de la musique et du cinéma qui sautent aux yeux, non pas les livres ni la littérature, au point que l'on pourrait parler à son sujet d'ethos 
littéraire dévoyé par le dispositif numérique, l'auctorialité se construisant de plus en plus ailleurs que dans le livre.

13 L'e-réputation brode donc en régime numérique en bien des points la même fable que celle construite par le marketing éditorial en régime médiatique (Ducas 2016) : le corps glorieux (visage et voix) comme insigne, le nom d'auteur comme signature, les livres comme oripeaux, et le récit de soi en écrivain comme posture.

\section{Ethos biographique et storytelling}

14 Car en tension avec ces stigmates promotionnels, le site d'écrivain est aussi un geste d'accueil et d'hospitalité qui invite l'internaute à entrer dans un univers singulier, dans une démarche qui garde malgré tout quelques vestiges de la pratique sociale de la visite au grand écrivain en ce que la rencontre avec lui tient d'un simulacre de visite guidée de son cabinet de travail. À ce titre, aucune interactivité n'est suggérée sur aucun des trois sites. Mais pas un qui n'accompagne cette visite d'une signalétique propre à l'invite et au repérage - flèches, titraille, bandeaux, puces, tabulations, « signes passeurs » (Souchier, Jeanneret et Le Marec 2003) - ou d'une ergonomie conviviale (page d'accueil, hyperliens, contact, renvoi aux réseaux sociaux, etc.), avec ses stations et ses haltes (les rubriques plus ou moins constantes et interchangeables du menu selon le paradigme «l'homme et l'œuvre »). À une époque où l'écrivain n'est plus ce grand homme dont on célèbre le sacre et où les gloires médiatiques se conquièrent ailleurs que sur la scène de l'écriture, il est sommé, pour retenir l'attention de l'internaute, de se mettre en scène et de parler de lui, et d'exister dans le présent de l'actualité littéraire.

D'où l'agenda rigoureusement tenu des conférences, lectures, rencontres, dans une dynamique temporelle qui, dans un feuilleté complexe de strates, va des archives conservées, classées et scrupuleusement datées, aux rendez-vous à venir à ne pas manquer. Car l'effet charismatique de l'écrivain repose moins désormais sur l'écriture que sur sa personne et celle-ci se met en scène selon le rituel d'une révélation photographique plus ou moins fournie (véritable album photos pour Chloé Delaume, rares images volées pour Éric Chevillard) et unifiée (même photo sur son site et sur Facebook pour Régine Detambel, dont une variante est téléchargeable sur son site), parfois même d'une voix se faisant entendre via un podcast audio (Régine Detambel) ou vidéo (Éric Chevillard, Chloé Delaume). Face à une industrie du livre pressée dans laquelle la rotation des titres est de plus en plus rapide, et d'une critique médiatique débordée qui ne s'arrête pas nécessairement sur eux, les livres ne suffisent pas à ce que l'écrivain gagne en visibilité et acquiert de la valeur. Pire : le capital symbolique de la littérature a diminué (Villemain 2009), le statut auctorial s'est dégradé, le livre est de plus en plus menacé par d'autres bien culturels. Pourtant, sur les sites étudiés, c'est bien encore sur les livres que se construisent les postures auctoriales, que l'on soit « auteur Azerty », performeuse ou bibliothécaire / camelot. En ce sens, on peut dire que le site d'écrivain est ce lieu de résistance du livre et de postures qui se font littéraires ou artistiques grâce à eux. D'où la présence en nombre sur tous les sites étudiés des ouvrages dont les visuels de couverture sont présentés en facing comme dans une librairie, mais aussi des critiques médiatiques et universitaires recensées comme autant d'indices de légitimation littéraire.

S'ils font la part belle aux livres, les sites - et avec eux, les ethe auctoriaux qui s'y dessinent - n'en sont pas moins aliénés à une nécessité inhérente à l'espace labyrinthique et foisonnant de la blogosphère et du Web 2.0 : celle de mobiliser une scénographie de 
l'attention - qui est aussi une économie (Citton 2014b) -, à même de faire face à la pléthore éditoriale et d'éviter le déficit attentionnel, à l'heure de la googlisation des esprits, de la surabondance des biens de consommation y compris culturels et du déferlement d'images et d'informations dans l'univers numérique. Si l'attention est bien «la ressource cruciale de notre époque » (Citton 2014a), précisément parce qu'elle est devenue rare, tant elle s'éparpille et peine à se concentrer, de fait, le site d'écrivain vise à capter l'attention, et pour y parvenir, il mise sur un procédé : le storytelling. Car tout autant, sinon plus que celle, évidente, de la visibilité, notre époque est bien celle de la narrativité. Jamais on n'a autant parlé et créé du lien social en communiquant autour d'histoires à partager collectivement. Le marketing a importé depuis longtemps ce modèle américain et fait du storytelling une «arme de distraction massive " incontournable (Salmon 2007 : 157) à laquelle n'échappent pas les sites d'écrivains. Or le storytelling agit sur la posture auctoriale en ce que les écrivains s'en emparent différemment.

Refuser de parler de soi est sans doute la pratique la plus judicieuse du storytelling. C'est la posture adoptée par Éric Chevillard. Elle est visible dès la notice biographique totalement burlesque imitant comme un écho sonore le style de l'auteur :

Éric Chevillard, né un 18 juin à la Roche-sur-Yon, anciennement Napoléon-Vendée, il ne s'endort pas pour autant sur ses lauriers puisqu'on le voit encore effectuer bravement ses premiers pas cours Cambronne, à Nantes. Il a deux ans lorsqu'il met un terme à sa carrière de héros national. Il brise alors son sabre sur son genou puis raconte à sa mère qu'il s'est écorché en tombant de cette balançoire et elle feint gentiment de le croire.

Cette posture de l'esquive biographique, l'introduction à l'entretien pour le site prixvirilo.com ${ }^{9}$ la résume parfaitement :

Éric Chevillard n'aime pas trop se la raconter. On comprend sa démarche : papoter de son œuvre, c'est soit du marketing littéraire (un oxymoron), soit un commentaire de texte qui s'adresse à ceux qui ne savent pas vous lire... Dès lors, l'éventualité d'un bon gros ramdam s'éloigne cruellement.

Ces considérations font d'Éric Chevillard un médiatique taiseux et bourru, genre Paparemborde mais moustache rasée. Impression renforcée par le fait qu'il vive à Dijon, ce qui est so terroir.

Et Éric Chevillard de conclure cet entretien - avec humour et connivence pour le site d'un prix iconoclaste et frondeur, mais quand même -, "j’ai une œuvre à écrire ", syntagme par excellence de l'écrivain qui ne quitte jamais la sphère de l'écriture et de l'œuvre en train de s'écrire. De fait, tous ses livres, qui détournent les codes narcissiques de l'autofictif et proposent dans des fictions joueuses des synopsis romanesques de l'auteur, du bavard délirant au misanthrope atrabilaire, sont à la fois l'origine et le prolongement naturel d'un site qui entretient ce pacte de lecture. Il y cultive cette posture éminemment littéraire, et se conforme en cela globalement (car la photo parfois résiste et incarne malgré tout, çà et là, l'écrivain) à l'image d'un «auteur Minuit » s'inscrivant dans la topique moderne de la mort de l'auteur, qui s'efface en tant que personne dans un « tissu de citations » (Barthes [1968] 2002 : 43).

La posture de Chloé Delaume est, elle, d'une autre nature: le but est d'injecter de l'autofiction partout où le réel construit du biographique pour celle qui se désigne désormais comme cette "Sibylle nomade, mais toujours connectée » et dit s'appeler " généralement Chloé Delaume, parce que je suis un personnage de fiction ${ }^{10}$ ». Pour ses lecteurs, ce sésame narratif résonne comme un effet de connivence car il renvoie à 
nombre de ses textes, depuis le tout premier, Le Cri du sablier, jusqu'à Corpus Simsi, en passant par toutes les performances menées par l'écrivaine, notamment celle, récente, des "Sorcières de la République ", "une performance, une autofiction collective, une narration grandeur nature », "une fiction fabriquée dans le réel ${ }^{11}$ ». Sur ce sésame, se fonde une posture artistique dont le but est de déréaliser un Moi biographique qu'il s'agit de corriger en effaçant toutes les ecchymoses d'un vécu invérifiable et de convertir en un Moi transpersonnel apte aux expérimentations collectives, ce que reflète parfaitement aussi le site, tout en étant une autre manière habile de ne pas parler de soi selon les codes autobiographiques ou "people» traditionnels. De fait, le découpage de la notice biographique ${ }^{12}$ en "saisons " cinématographiques agrémentées d'une série de dessins tirés du premier site de l'écrivaine ne fait pas oublier ce qu'offre la nouvelle ergonomie du site : une incarnation auctoriale dans le virtuel, qui théâtralise l'écrivaine en habits de performeuse, optimise le potentiel de scénographies qui n'ont pas que l'encre des mots comme outil, mais aussi la voix, la vidéo, la scène théâtrale, les pièces sonores et les chansons. "S'écrire par-delà le papier ${ }^{13}$ " revient à se donner à voir et à entendre, à ce que site, voix, corps et écriture (puisque toute performance est « un livre en train de s'écrire » et un "roman en cours d'écriture ») communient dans une même idée de «l'autofiction [comme] pratique ${ }^{14} »$ où le virtuel fonctionne comme une véritable turbine à autofiction. Création artistique et image publique forment ainsi une seule et même "performance ", et d'une autre façon que Éric Chevillard mais avec la même force de conviction, tout n'est donc qu'art et littérature.

Régine Detambel, quant à elle, dans un dédoublement apparent que trahit la bipolarité "écrire/animer » de son portail, affiche une image d'écrivaine professionnelle ou plus précisément un portrait de l'auteur en médiatrice bibliothécaire. Pour celle qui fut d'abord kinésithérapeute, le paradigme du passeur de livres ${ }^{15}$ s'enrichit d'une dimension de formatrice à la curative "bibliothérapie créative ${ }^{16}$ » qui envahit une large partie du site - tout comme sa page personnelle sur Facebook - et concurrence l'image d'une écrivaine prolixe, auteure de plus de 50 livres. "Les livres prennent soin de nous " résonne comme un slogan que l'on retrouve aussi bien dans l'onglet "Actualités", puisqu'il s'agit aussi du titre d'un livre venant de paraitre aux éditions Actes Sud (couverture et "pitch » dupliqués à plusieurs endroits du site) dont on assure du même coup la promotion, que dans les onglets "Espace bibliothécaire » et "Bibliothérapie ». Conférences, journées ou stages de formation tarifés incitent, dans une démarche fortement injonctive $\mathrm{e}^{17}$, les professionnels des bibliothèques à ouvrir leur propre atelier de bibliothérapie et à le faire grâce à l'auteure. Dans un discours qui tient de la croisade littéraire ou de la mission mystique comme du cri du camelot ou du colporteur pour celle qui, sans doute, vit de ses ateliers de bibliothérapie et animations en médiathèques plus que de sa plume, l'auteure appelle les professionnels du livre à s'emparer de cette discipline anglo-saxonne pour la mettre au service de « la vraie littérature que nous nous devons de défendre ${ }^{18}$ ", menacée selon elle par les livres de développement durable auxquels cette même discipline initie. L'onglet «Bibliothérapie » redouble ce discours, publicitaire à trop se vouloir militant, d'une « exploration des vertus thérapeutiques de la lecture et de l'écriture ${ }^{19}$ ». À plusieurs reprises, la littérature est mobilisée comme cet espace thérapeutique où « les médecins se nomment Ernest Hemingway, Colette, Camille Laurens et Kenzaburô Oé et leur ordonnance se lit sur des centaines de pages, sans prescription de doses. La littérature est un remède universel ». La posture littéraire ne se dément pas, mais tente de redoubler cette mission médiatrice : dans un entretien ${ }^{20}$ qui la résume mais dont l'accès n'est pas immédiat, à l'injonction gracquienne en lisant, en 
écrivant, s'ajoute l'« éloge d'une lecture caressante de la paume de la main sur le papier du livre », de " la peau du papier » et du « corps du livre », d'une « sensibilité de l'écriture » qui est «transitive et non réflexive", se construit une image d'écrivaine faisant de la littérature son fonds de commerce, que ne contredit pas sa mission de médiatrice, mais que le dispositif numérique étouffe sous une communication d'un ethos bibliothécaire écrasant.

\section{Ethos numérique et métamorphoses du geste auctorial}

Les postures auctoriales, pour exister en régime numérique, démultiplient donc les marques de littérarité, mais pas toujours au service de l'ethos auctorial qu'on souhaiterait convoquer. Régine Detambel, par exemple, cite à foison Pierre Guyotat, ce grand écrivain pourtant totalement étranger à son univers d'écriture. Rien d'étonnant dès lors si ces postures se fondent sur une métatextualité et une intertextualité fortes, dans lesquelles on peut lire la tension tangible entre deux régimes de présentation de soi, un ethos d'écrivain (celui qui écrit une œuvre) et un ethos d'auteur (celui qui publie chez des éditeurs des livres dont sa signature le porte garant). Même pour des écrivains comme Éric Chevillard et Chloé Delaume, dont les textes cultivent une forte intertextualité et dont la pratique du pastiche et du détournement parodique est constitutive de l'écriture, pas un entretien mis en ligne sur leurs sites qui ne démultiplie, du coup, les références et les filiations littéraires. Face à la communauté interprétative des internautes et des lecteurs, l'écrivain se doit d'abord d'exister au sein d'une communauté littéraire, passée ou présente. "Hors le texte, le texte » (Rabau 2002: 21) et perdure ainsi la posture et l'imaginaire de l'écrivain inspiré et cultivé qui, de manière très attendue, cite ses pairs et ses modèles en écriture, voire même pose en " auteur en réseau ", connecté à différents espaces de sociabilité littéraires ou artistiques. De ce point de vue, Chloé Delaume est la plus inventive : l'onglet «Performances \& Cie » énumère ses collaborateurs et «amis » qui s'affichent déjà sur sa page Facebook; et sur Twitter, quand elle ne parle pas de ses insomnies et de son chat Temesta Delaume, elle fait de la veille et retweete beaucoup d'informations sur des poètes, plasticiens, performers, écrivains qu'elle aime (Antoine Volodine, Lydie Salvayre, etc.), des festivals (les Utopiales, dont elle est membre du jury), conseille des livres, des films, tisse du réseau culturel et intellectuel.

Mais le dispositif numérique trouve surtout sa singularité médiatique à autoriser et favoriser des formes d'intratextualité. Autocitation (Éric Chevillard et son crabe ; Chloé Delaume et son " personnage de fiction»), redondance citationnelle (Régine Detambel et la sortie de son dernier livre; Chloé Delaume et sa performance collective des « Sorcières de la République »), qui non seulement consolident les contours d'un univers littéraire singulier, mais incarnent l'ethos, lui donnent de la chair et de la voix. On se souvient comment le peintre Pierre Bonnard parlait de son art : «Il ne s'agit pas de peindre la vie, il s'agit de rendre vivante la peinture. » D'une façon voisine, pour l'écrivain en son site, il ne s'agit pas de se peindre, il s'agit de rendre vivante l'image de soi, autrement dit de produire un ethos incarné, vivant. Et la façon la plus «numérique » d'y parvenir, c'est de convertir la vitrine de l'œuvre déjà réalisée, publiée, en geste de créer ou d'écrire. La trace et le geste: le geste se fait encyclopédique («Le grand élucidaire» de Régine Detambel, cette encyclopédie où l'auteure "serre ses gloses » dans la lignée d'un Michel Leiris), archivistique (Chloé Delaume archivant ses «news"; Éric Chevillard archivant son blog), poétique (les billets de Régine Detambel, les posts du blog d'Éric Chevillard). 
L'œuvre s'y fait work in progress, écriture en devenir, à venir, jamais figée, avec ses effets d'annonce, ses "à paraître", ses projets consignés pour dessiner un demain de la création, une pérennité du geste d'écrire et l'impression d'une écriture immédiate en train de s'écrire.

Soumis à ce flux d'écriture que le site ou le blog déverse, l'écrivain se donne à voir en habits de polygraphe boulimique, d'artisan de l'écriture ouvert aux quatre vents : on y découvre un Éric Chevillard dont la figure polymorphe déborde celle de l'«auteur Minuit ", écrivant pour les enfants, préfacier, blogueur, critique littéraire tenant le feuilleton hebdomadaire du Monde des livres depuis 2011, collaborant avec des illustrateurs (Philippe Favier chez Fata Morgana) ; une Régine Detambel "tout-terrain » écrivant pour la jeunesse, essayiste, auteure de livres numériques, de livres d'artistes, participant à des ouvrages collectifs médicaux, poétiques, artistiques, régionaux, animant des ateliers d'écriture et des cafés littéraires; une Chloé Delaume auteure de théâtre, de livres-jeux, d'essais, de fictions radiophoniques, à la fois écrivaine, éditrice, performeuse, blogueuse, chanteuse, réalisatrice, chroniqueuse et "forumancière ", bricoleuse multisupport, outils et techniques, collaborant avec des musiciens, poètes, plasticiens, designers. Portrait de l'artiste en artisan multitâche dont le site, relayé par les réseaux sociaux, consigne toutes les formes, enregistre tous les avatars et les métamorphoses, au point de noyer un peu la figure d'écrivaine, qu'elle revendique pourtant au premier plan. «L'expansion du domaine de la liste » qu'offre le site dans ses feuilletés et ses déroulés, ses arborescences et sa géographie labile, participe de cette représentation d'un écrivain professionnel et non plus en chambre, mais surtout créateur compulsif et hyperactif, débordé et débordant d'activités: liste des rendez-vous de son agenda, liste de ses publications, liste de ses éditeurs, liste de ses projets, liste de ses lectures... Cet espace de la liste dont Eco (2009) a montré combien il pouvait être l'énumération et l'inventaire pratiques et triviaux de ce qui est - les livres comme trophées de l'écrivain, en quelque sorte -, mais aussi suggérer le vertige de l'inventaire infini aux portes de l'immensité homérique (l'immensité de l'armée grecque dans L'Iliade) et du sublime kantien (la voûte du ciel étoilé) dont rêve l'écrivain à l'ombre de l'auteur.

Le site d'écrivain comme incubateur de littérature et "accélérateur de particules » littéraires : c'est sur ce point que j'aimerais conclure, tout en voyant en lui et dans les postures qu'il génère un indicateur fort de ce qui est en train de changer en matière d'imaginaire du littéraire, notamment de publication de l'écrivain.

L'ethos de l'écrivain sur son site illustre, en effet, la profonde métamorphose de la figure de l'auteur, qui n'est plus cette figure in absentia décrite par Derrida (2004) - l'écrivain en chambre, solitaire, à l'écritoire, radicalement absent, dont seul l'imprimé véhicule les textes à un public de masse, anonyme. Métamorphose de l'auteur, entré en visibilité non seulement médiatique mais numérique. Écrivain de proximité comme on le dit d'un commerce, en somme, écrivain hors les murs, hors les « règles de l'art » bourdieusiennes opposant un pôle élitiste et un pôle de grande production désormais caduques, écrivain «médiaculturel » (Maigret et Macé 2006) et polygraphe. Écrivain dont les mises en scène numériques, situées à la frontière désormais poreuse de la création et de la vitrine publicitaire, mêlent promotion et création, et surtout débordent largement le cadre du livre ou de la publication papier pour dessiner un nouvel espace du littéraire fondé, comme l'a montré Jérôme Meizoz, sur une conduite, un discours et une poétique, ce que, précisément, on appelle la posture de l'auteur. 


\section{BIBLIOGRAPHIE}

Amossy, Ruth, 2006, L'Argumentation dans le discours, Paris, Armand Colin, coll. « cursus ».

Barthes, Roland, [1968] 2002, « La mort de l'auteur », dans CEuvres complètes, III (1968-1971), Paris, Seuil.

Beaudouin, Valérie, 2012, «Trajectoires et réseau des écrivains sur le Web : construction de la notoriété et du marché », Réseaux, n 175, p. 107-144.

Bon, François, 2011, Après le livre, Paris, Seuil.

Certeau, Michel de, [1980] 1990, L'Invention du quotidien, Paris, Gallimard.

Chapelain, Brigitte et Fort, Pierre-Louis, 2015, « Pratiques et représentations médiatiques des écrivains à l'heure des réseaux. De la promotion à de nouvelles écritures », dans A.-M. Petitjean et V. Houdart-Merot (dir.), Numérique et écriture littéraire, Paris, Hermann, p. 149-151.

Chartier, Roger, 2008, Écouter les morts avec les yeux, Paris, Collège de France, Fayard.

Chevillard, Éric, 1993, La Nébuleuse du crabe, Paris, Minuit.

Chevillard, Éric, 2014, Le Désordre Azerty, Paris, Minuit.

Citton, Yves, 2014a, Pour une écologie de l'attention, Paris, Seuil, coll. « La Couleur des idées ».

Citton, Yves, 2014b, L'Économie de l'attention, nouvel horizon du capitalisme ?, Paris, La Découverte.

Derrida, Jacques, 2004, La Carte postale de Socrate à Freud et au-delà, Paris, Flammarion.

Deseilligny, Oriane, 2015, «Tiers livre : entre auctorialité et architextualité, jeux d'écritures en régime numérique », dans Tiers Livre dépouille \& création, Komodo 21, 1, http://komodo21.fr/tierslivre-entre-auctorialite-architextualite-jeux-decriture-regime-numerique/, consulté le 25 février 2016.

Deseilligny, Oriane et Ducas, Sylvie (dir.), 2013, L'Auteur en réseau, les réseaux de l'auteur, Nanterre, Presses universitaires de Paris Ouest.

Ducas, Sylvie, 2010, « Fiction auctoriale, posture et impostures médiatiques : le cas de Chloé Delaume, "personnage de fiction" ", Temps et Médias, nº 14, p. 176-192.

Ducas, Sylvie, 2016 (à paraître), « L'écrivain contemporain entre marque et label, branding et storytelling : la littérature à quel(s) prix ? ", dans M.-È. Thérenty et A. Wrona (dir.), L'Écrivain comme marque, Paris, Presses de l'Université Paris-Sorbonne.

Eco, Umberto, 2009, Vertige de la liste, Paris, Flammarion.

Genette, Gérard, 2002, Seuils, Paris, Seuil. 
Guilet, Anaïs, 2010, « Lire le jeu vidéo, jouer à la littérature : Corpus Simsi de Chloé Delaume », dans S. Craipeau, S. Genvo et B. Simonnot (dir.), Questions de communication, série acte $8:$ Les jeux vidéo au croisement du social, de l'art et de la culture, Nancy, Presses universitaires de Nancy, p. 223-236.

Le Guern, Philippe (dir.), 2002, Les Cultes médiatiques. Culture fan et œuvres cultes, Rennes, Presses universitaires de Rennes.

Maigret, Éric et Macé, Éric (dir.), 2006, Penser les médiacultures. Nouvelles pratiques et nouvelles approches de la représentation du monde, Paris, Armand Colin.

Maingueneau, Dominique, 2002, «Problèmes d'ethos », Pratiques, n 113-114, p. 55-67.

Méadel, Cécile et Sonnac, Nathalie, 2012a, L'Auteur au temps du numérique, Paris, Labs Hadopi/ Éditions des archives contemporaines.

Méadel, Cécile et Sonnac, Nathalie, 2012b, « L'auteur au temps du numérique », Esprit, $\mathrm{n}^{\circ}$ 5, p. 102-114.

Meizoz, Jérôme, 2007, Postures littéraires. Mises en scène modernes de l'auteur, Genève, Slatkine. Meizoz, Jérôme, 2009, «Ce que l'on fait dire au silence : posture, ethos, image d'auteur », Argumentation et analyse du discours, $\mathrm{n}^{\circ} 3$, https://aad.revues.org/667, consulté le 29 février 2016.

Nora, Olivier, 1986, «La visite au grand écrivain », dans P. Nora (dir.), Les Lieux de mémoire, II, La Nation, Paris, Gallimard, p. 563-585.

Peras, Delphine, 2012, «Les gazouillis de Twitter sont-ils dignes des écrivains ? », L'Express.fr, 18 juillet 2012, http://www.lexpress.fr/culture/livre/les-gazouillis-de-twitter-sont-ils-dignesdes-ecrivains_1139270.html, consulté le 29 février 2016.

Rabau, Sophie, 2002, L’Intertextualité, Paris, GF Flammarion.

Salmon, Christian, 2007, Storytelling, la machine à fabriquer des histoires et à formater les esprits, Paris, La Découverte, coll. «Poche».

Souchier, Emmanuël, Jeanneret, Yves et Le Marec Joëlle (dir.), 2003, Lire, écrire, récrire. Objets, signes et pratiques des médias informatisés, Paris, BPI.

Villemain, Marc, 2009, entretien avec Éric Bonnargent, « Littérature et internet », BSC news magazine, $\mathrm{n}^{\circ}$ 15, http://www.marcvillemain.com/archives/2009/04/25/13490998.html, consulté le 29 février 2016.

\section{NOTES}

1. "Écouter les morts avec les yeux ", titre de l'ouvrage de Roger Chartier compilant ses leçons inaugurales du Collège de France, Collège de France/Fayard, 2008.

2. Voir les actes de ce colloque dans Deseilligny et Ducas (2013).

3. L'étude s'appuie surtout sur leurs sites respectifs: www.chloedelaume.net; http:// www.detambel.com ; www.eric-chevillard.net, et le blog de ce dernier : http://l-autofictif.overblog.com, ainsi que sur les pages Facebook de Chloé Delaume et de Régine Detambel, et le compte Twitter de Chloé Delaume, https://twitter.com/chloe_delaume.

4. Nos analyses, principalement centrées sur le site d'écrivain, s'autoriseront néanmoins des incursions du côté des réseaux sociaux et de Google et Wikipédia.

5. Voir l'évocation du premier de ces sites dans notre article (2010).

6. En référence au roman d'Éric Chevillard (2014), Le Désordre Azerty, Paris, Minuit. 
7. En bas de la page d'accueil, elle s'amuse de l'environnement technologique et ironise sur un site « fièrement propulsé par Wordpress ».

8. «Écrivain et performeuse » sur le portail du site, "personnage de fiction pire que les autres» dans l'onglet autobiographique «Parcours », « écrivaine et Cie » sur Twitter, « auteur [...] née en 1973 dans un corps à l'identité enterrée depuis [et] devenue un personnage de fiction s'écrivant lui-même » sur Facebook, « écrivaine française » sur Wikipédia, « écrivaine » dans le rich snippet de Google.

9. "Éric Chevillard: la rencontre. Dix questions engagées à Éric Chevillard", prixvirilo.com, 22 novembre 2011.

10. Page d'accueil de son site.

11. Ibid.

12. Onglet « Parcours » : http://www.chloedelaume.net/?page_id=105.

13. «S'écrire par-delà le papier: hybridation des formes et de supports dans l'œuvre autofictionnelle de Chloé Delaume ", journée d'étude organisée par Florence Thérond et Annie Pibarot, Université de Montpellier, 5 novembre 2014.

14. Onglet « Parcours », saison 7, «L'autofiction est une pratique ».

15. Voir la rubrique « lisotter » dans le menu de l'onglet «Écrire ».

16. Le rich snippet de Google la désigne d'abord comme kinésithérapeute de formation et bibliothérapeute avant de dire qu'elle est une auteure.

17. Participe de cette injonction la démultiplication des lieux où figurent les coordonnées personnelles (numéro de mobile) et ses adresses de messagerie.

18. Onglet « Espace bibliothécaire ».

19. Onglet «Bibliothérapie ».

20. On en trouve le pdf dans l'onglet « L'auteur » présent sur la page d'accueil.

\section{RÉSUMÉS}

Cet article se propose d'interroger trois démarches très différentes d'écrivains utilisant leur site web comme espace de construction d'un ethos auctorial inséparable d'un usage très spécifique du Web 2.0. L'atelier d'écriture d'Éric Chevillard, l'espace performatif de Chloé Delaume et la vitrine publicitaire de Régine Detambel dessinent, en effet, des postures variées de l'écrivain au travail. Si le livre et la bibliothèque continuent d'y fonder la posture auctoriale et le geste d'écrire, l'écrivain s'y raconte et s'y rend public d'une façon nouvelle, induite par les dispositifs numériques.

This article aims to examine three very different approaches of writers using their site as a building space for expressing their ethos inseparable from a very specific use of Web 2.0. Éric Chevillard's writing workshop, the performative space of Chloé Delaume and Régine Detambel's advertising showcase illustrate in fact, various postures of the writer at work. If the book and the library continue to found the authorial posture and writing gesture, the writer speaks about himself and makes himself public in a new way, driven by digital devices. 
INDEX

Mots-clés : site web, écrivain, posture, storytelling, publication

Keywords : website, writer, publishing

\section{AUTEUR}

SYLVIE DUCAS

Université Paris Ouest Nanterre La Défense 